\title{
Illness cognitions and family adjustment: psychometric properties of the Illness Cognition Questionnaire for parents of a child with cancer
}

\author{
Simone M. Sint Nicolaas ${ }^{1} \cdot$ Sasja A. Schepers $^{2}$ - Esther M. M. van den Bergh ${ }^{1}$. \\ Andrea W. M. Evers ${ }^{1,3}$ - Peter M. Hoogerbrugge ${ }^{4,5}$ - Martha A. Grootenhuis ${ }^{2}$. \\ Christianne M. Verhaak ${ }^{1}$
}

Received: 22 January 2015 / Accepted: 2 June 2015 /Published online: 25 June 2015

(C) The Author(s) 2015. This article is published with open access at Springerlink.com

\begin{abstract}
Purpose Illness cognitions are an important mediator between disease and psychological adjustment. This study assessed the psychometric properties of the Illness Cognition Questionnaire (ICQ), adjusted for the parents of an ill child.

Methods Participants were recruited from two multicenter studies: sample 1 included 128 parents of a child diagnosed with acute lymphoblastic leukemia (ALL) (response rate $82 \%$ ) and sample 2 included 114 parents of a child diagnosed with cancer (response rate $74 \%$ ). Parents completed an adapted version of the ICQ (Illness Cognition Questionnaire-Parent version (ICQ-P)), together with the Profile of Mood States (POMS; sample 1) or the Hospital Anxiety and Depression Scale (HADS; sample 2). The factor structure of the ICQ-P was examined by means of principal component analysis. Cronbach's alpha for each subscale and correlations between the ICQ-P scales and the HADS and POMS were calculated. The illness cognitions of parents with and without psychological distress were compared.
\end{abstract}

Simone M. Sint Nicolaas

simone.sintnicolaas@radboudumc.nl

1 Department of Medical Psychology, Radboud University Medical Center, Nijmegen, The Netherlands

2 Psychosocial Department, Emma Children's Hospital Academic Medical Center, Amsterdam, The Netherlands

3 Health, Medical and Neuropsychology Unit, Leiden University, Leiden, The Netherlands

4 Pediatric Oncology, Radboud University Medical Center, Nijmegen, The Netherlands

5 Dutch Childhood Oncology Group (SKION), The Hague, The Netherlands
Results Factor analysis confirmed the hypothesized structure of the ICQ-P in our sample $(n=242)$. The three scales Helplessness, Acceptance, and Perceived Benefits explained $9.8,31.4$, and $17.9 \%$ of the variance, respectively. Cronbach's alpha showed adequate internal consistency (.80-.88). Concurrent and criterion-related validity were appropriate.

Conclusions The results confirm that the ICQ-P reliably assesses the illness cognitions of the parents of a child with cancer. Psychologically distressed parents showed less acceptance and more helplessness. The availability of a short and valid illness cognition questionnaire will help clinicians gain insight into parental cognitions regarding the illness of their child, information that might be helpful for targeting interventions.

Keywords Illness cognition questionnaire - Validation · Parents · Childhood cancer

\section{Introduction}

Patients diagnosed with a chronic illness have their own beliefs about their illness, defined as illness cognitions. Illness cognitions can be described as a patient's perception, interpretation, and understanding of the disease and its treatment [1-3]. Illness cognitions refer to the common-sense model of illness representations from Leventhal [1]. The theory describes beliefs and expectations people have regarding a disease or medical complaints. A patient's beliefs influence their ability to cope with and adjust to illness [4], and illness cognitions may be a significant mediator between the condition and the patient's well-being [5-9]. For example, patients who 
perceive their illness as having serious consequences and as being chronic, experience more physical, emotional, and social problems than do patients who perceive their illness as being curable and controllable [6-8].

Similar findings have been obtained regarding the illness cognitions of the parents of an ill child, particularly a child with cancer. Parental cognitions about how stressful the illness is to the child, how life-threatening the cancer is, the intensity of treatment, and their own ability to cope with their child's disease are significantly associated with parental distress [10-13]. Parents who are optimistic and who see benefits are less distressed than parents who do not have this optimistic frame of mind [14-16]. In turn, parental distress influences the child's distress [17-21], and therefore illness beliefs affect the psychological adjustment of the entire family. Insight into parental cognitions regarding their child's disease may help therapists to understand maladaptive adjustment. While there are some questionnaires to assess parental illness cognitions $[12,22]$, they mainly focus on negative illness cognitions. We used the Illness Cognition Questionnaire (ICQ) in this study because it has been shown to predict adjustment problems in adults with chronic conditions [5, 23] and includes both positive and negative cognitions related to disease, namely, helplessness, acceptance, and perceived benefits.

The objectives of this study were to adapt the ICQ, which was originally developed for adults with a chronic condition [5], for use in parents of an ill child (Illness Cognition Questionnaire-Parent version (ICQ-P)), to assess its psychometric properties, and to determine whether scores are associated with parental distress. We also investigated whether the original three-factor structure (Helplessness, Acceptance, and Perceived Benefits) found in adult patients is equally valid for the parents of children with cancer. We expected that the ICQ$\mathrm{P}$ would have an adequate factor structure and appropriate reliability and validity in our sample. Furthermore, we expected adaptive illness cognitions (Acceptance) to be negatively associated with parental psychological distress and hypothesized that maladaptive illness cognitions (Helplessness) would be associated with parental psychological distress.

\section{Methods}

\section{Sample}

Data on the illness cognitions of the parents of a child with cancer were collected in two studies: sample 1 consisted of parents who participated in the period 2006-2009 and sample 2 consisted of parents who participated in the period 20122013. The two studies were similar in terms of including the parents of a child diagnosed with cancer and the time since diagnosis (around 1 month, see Table 1). Families in sample 1 completed paper-and-pencil questionnaires, whereas families in sample 2 filled in Web-based questionnaires.

The first sample comprised the parents of children with acute lymphoblastic leukemia (ALL) recruited from six of the seven Dutch pediatric oncology centers (response rate $82 \%$ ) [24]. Reasons for non-response were too overwhelmed or medical complications. This study focused on the adjustment of children with ALL during treatment and predictors of child adjustment. Inclusion criteria were age 1.5-18 years, recent diagnosis of ALL, treatment according to the Dutch Childhood Oncology Group (DCOG) ALL 10 protocol [25], and parental fluency in Dutch. The study was approved by the medical ethics review boards of the participating institutions.

The second sample comprised the parents of children recently diagnosed with cancer from four of seven Dutch pediatric oncology centers (response rate $74 \%$ ). Reasons for nonresponse were too overwhelmed, not interested, or medical complications. This study focused on the validation of the Psychosocial Assessment Tool (PAT) and investigated the effects of psychosocial screening in pediatric oncology. Inclusion criteria were age $0-17$ years, recent diagnosis of cancer, and parental fluency in Dutch; children with a life expectancy $<6$ months or a relapse were excluded. The study was approved by the medical ethics review boards of the participating institutions.

The samples were compared regarding child age and gender, and time since diagnosis. The patients in sample 1 were younger $(T=-2.25, p=.03)$, and their parents completed the questionnaires longer after diagnosis $(T=4.30, p=.00)$ than the patients/parents in sample 2 . Patient gender was not significantly different $\left(\chi^{2}=.78, p=.38\right)$.

\section{Measures}

Sociodemographic information (diagnosis, family structure, socioeconomic status) was collected with a self-developed questionnaire.

Parental illness cognitions about the disease of their child were assessed with the ICQ-P (adapted from the ICQ with permission from the developers) [26]. See "Appendix" for the questionnaire. The ICQ measures illness cognitions that reflect different ways of evaluating the aversive character of a chronic condition of a patient, namely, helplessness (e.g., "My illness controls my life"), acceptance (e.g., "I can handle the problems related to my illness"), and disease benefits (e.g., "Dealing with my illness has made me a stronger person"). Items are scored on a 4-point Likert scale ( $1=$ not at all, $2=$ somewhat, $3=$ to a large extent, $4=$ completely), and each subscale consists of 6 items. Scale scores are calculated by summing the item scores, resulting in a subscale score ranging from 6 to 24 and a total score ranging from 18 to 72 . The text of the questions was adapted to make it appropriate for the parents of ill children. For example, "my illness" in the 
Table 1 Demographic information of participating families

\begin{tabular}{|c|c|c|c|}
\hline & $\begin{array}{l}\text { Study } 1 \\
N=128\end{array}$ & $\begin{array}{l}\text { Study } 2 \\
N=114\end{array}$ & $\begin{array}{l}\text { Total } \\
N=242\end{array}$ \\
\hline \multicolumn{4}{|l|}{ Age parent } \\
\hline$M(\mathrm{SD})$ & $38.20(5.99)$ & $39.86(6.55)$ & $38.98(6.30)$ \\
\hline Range & $25-55$ & $24-55$ & $24-55$ \\
\hline \multicolumn{4}{|l|}{ Gender parent } \\
\hline Mother & $111(87 \%)$ & $74(65 \%)$ & $185(76 \%)$ \\
\hline Father & $17(13 \%)$ & $40(35 \%)$ & $57(24 \%)$ \\
\hline \multicolumn{4}{|l|}{ Education parent } \\
\hline Low & $16(13 \%)$ & $9(8 \%)$ & $25(10 \%)$ \\
\hline Medium & $62(48 \%)$ & $37(32 \%)$ & $99(41 \%)$ \\
\hline High & $50(39 \%)$ & $68(60 \%)$ & $118(49 \%)$ \\
\hline \multicolumn{4}{|l|}{ Marital status parent } \\
\hline Single & $15(12 \%)$ & $3(3 \%)$ & $18(7 \%)$ \\
\hline \multicolumn{4}{|l|}{ Age child } \\
\hline$M(\mathrm{SD})$ & $6.52(4.26)$ & $7.87(5.10)$ & $7.15(4.71)$ \\
\hline Range & $1-17$ & $0-17$ & $0-17$ \\
\hline \multicolumn{4}{|l|}{ Gender child } \\
\hline Boy & $68(53 \%)$ & $65(57 \%)$ & $133(55 \%)$ \\
\hline Girl & $60(47 \%)$ & $49(43 \%)$ & $109(45 \%)$ \\
\hline \multicolumn{4}{|l|}{ Diagnosis } \\
\hline \multicolumn{4}{|l|}{ Hematological } \\
\hline Leukemia & $128(100 \%)$ & $29(25.4 \%)$ & $157(64.9 \%)$ \\
\hline Hodgkin's lymphoma & & $8(7.0 \%)$ & $8(3.3 \%)$ \\
\hline Non-Hodgkin lymphoma & & $13(11.4 \%)$ & $13(5.4 \%)$ \\
\hline \multicolumn{4}{|l|}{ Neuro-oncological } \\
\hline Brain/CNS tumor & & $19(16.7 \%)$ & $19(7.9 \%)$ \\
\hline \multicolumn{4}{|l|}{ Solid } \\
\hline Ewing's sarcoma & & $6(5.3 \%)$ & $6(2.5 \%)$ \\
\hline Neuroblastoma & & $7(6.1 \%)$ & $7(2.9 \%)$ \\
\hline Rhabdomyosarcoma & & $6(5.3 \%)$ & $6(2.5 \%)$ \\
\hline Wilms' tumor & & $8(7.0 \%)$ & $8(3.3 \%)$ \\
\hline Osteosarcoma & & $11(9.6 \%)$ & $11(4.5 \%)$ \\
\hline Other solid tumor & & $7(6.2 \%)$ & $7(2.9 \%)$ \\
\hline \multicolumn{4}{|l|}{ Mean time after diagnosis } \\
\hline$M(\mathrm{SD})$ & 41 days (22.84) & 30 days (14.61) & 36 days $(20.08)$ \\
\hline Range & 5-131 days & 4-80 days & 4-131 days \\
\hline
\end{tabular}

original questionnaire was changed to "my child's illness". The internal consistency of the three scales of the original ICQ ranged from $\alpha=.84$ to $\alpha=.91$ [5].

Parental psychological distress (sample 1) was assessed with the Dutch shortened version of the Profile of Mood States (POMS) [27, 28]. The POMS is a self-report questionnaire investigating changeable mood states and consists of 32 items. It is designed to measure mood in five different domains: fatigue (6 items), irritation (7 items), vigor (5 items), tension (6 items), and depression ( 8 items). The answers are scored on a 5-point scale ranging from "not at all" (0) to "extremely" (4). The reliability and validity of this scale are good $(\alpha=.76-\alpha=.95)$ [27]. In the current study, internal consistency for the different domains ranged from $\alpha=.79$ to $\alpha=.91$.

Parental psychological distress (sample 2) was assessed with the Hospital Anxiety and Depression Scale (HADS) $[29,30]$. The HADS is a self-report questionnaire assessing the presence of anxious and depressive states in a medical setting. It consists of 14 items in two domains: anxiety (7 items) and depression (7 items). Answers are scored on a 4- 
point scale, ranging from 0 to 3 . The total score is an indication of overall distress (range 0-42). In this study, a cutoff score of $\geq 13$ for the total scale was used to distinguish clinically distressed parents from normally functioning parents [31]. The reliability and validity of this scale are good $(\alpha=.71-\alpha=.90)$ [30]. In the current study, internal consistency for the different scales ranged from $\alpha=.82$ to $\alpha=.91$.

\section{Statistical methods}

First, the factor structure of the ICQ-P was examined using principal component analysis (PCA) with oblique rotation with a fixed number of three factors, using Statistical Package for Social Sciences (SPSS) version 20. Analyses were first performed separately for the two samples, but results are reported for the combined sample because of the high levels of agreement. Kaiser-Meyer-Olkin Measure of Sampling Adequacy (KMO) and Bartlett's Test of Sphericity were checked before interpreting the rotated factor loadings. Kaiser recommends a minimum KMO of 0.5 [32]. Each item was assumed to load on one factor only. Factor loadings of 0.36 or higher were considered significant, based on a sample size of 200 [33]. To examine the psychometric properties of the ICQ-P, Cronbach's alpha was calculated for each subscale; a value of 0.60 or higher was considered acceptable [34]. Pearson correlations were calculated between the three subscales to investigate their mutual relationship. A one-way ANOVA was used to compare scores on the ICQ-P between mothers and fathers and between the different diagnoses (hematological=leukemia and lymphoma; solid=Ewing's sarcoma, neuroblastoma, rhabdomyosarcoma, Wilms' tumor, osteosarcoma, and other solid tumors; neurooncological=brain/CNS tumor). To test the concurrent validity of the ICQ-P, Pearson correlations between ICQ-P scores and POMS (sample 1) and HADS (sample 2) scores were calculated. To test criterion-related validity, the ICQ-P scores of distressed parents (HADS total score $\geq 13$ ) and non-distressed parents (HADS total score $\leq 12$ ) were compared, using a one-way ANOVA. This analysis was performed only with the HADS, which has a validated cutoff point, unlike the POMS. Cohen's $d$ was calculated as a measure of effect size. $P$ values $\leq 0.05$ were considered statistically significant. Effect sizes .20 were considered small, .50 medium, and .80 large [35].

\section{Results}

\section{Sample characteristics}

In total, there were 242 participants (Table 1). The patients were aged $0-17$ years $(M=7.15, \mathrm{SD}=4.71), 133$ boys $(55.0 \%)$ and 109 girls $(45.0 \%)$. The mean time from diagnosis to completion of the questionnaires was 36 days. Overall, $73.6 \%$ children were diagnosed with a hematological tumor, $7.9 \%$ with a neuro-oncological tumor, and $18.6 \%$ with a solid tumor. Questionnaires were completed by either mothers or fathers: 185 (76.4\%) mothers and $57(23.6 \%)$ fathers, aged 24 55 years $(M=38.98, \mathrm{SD}=6.30)$.

\section{Factor analysis and reliability of the ICQ-P}

The suitability of data for factor analysis was established with the Kaiser-Meyer-Olkin test (.87) and Bartlett's Test of Sphericity. The different items loaded on the three original factors (Helplessness, Acceptance, and Perceived Benefits) (Table 2). The three original subscales, each consisting of 6 items, explained 9.8, 31.4, and $17.9 \%$ variance, respectively. Together, this three-component solution explained $59.1 \%$ of the total variance. Cronbach's alpha (.80-.88) showed that the scales had an adequate internal consistency. The mean scores of mothers and fathers on all subscales were not significantly different, and scores did not differ by diagnosis. There was no correlation between the subscales Helplessness and Perceived Benefits $(r=-.04)$, a weak correlation between Acceptance and Perceived Benefits $(r=.28)$, and a moderate negative correlation between Helplessness and Acceptance $(r=-.48)$.

\section{Predicting parental psychological distress}

Concurrent validity was measured with Pearson's correlation coefficients between the ICQ-P and the measures of parental psychological well-being (Table 3). As expected, cognitions of Helplessness were moderately to relatively highly $(r=.42-.59)$ associated with a worse psychological well-being, that is, with higher levels of overall distress, depression, anxiety, tension, irritation, and fatigue, as assessed with the HADS and POMS. The opposite was seen for cognitions of Acceptance. Higher levels of Acceptance were moderately to relatively highly associated with a better psychological well-being, namely, higher levels of vigor and lower levels of overall distress, depression, anxiety, tension, irritation, and fatigue. No statistically significant correlations were found between the subscale Perceived Benefits and levels of psychological distress.

To test criterion-related validity, we compared the illness cognitions of parents who were clinically distressed (HADS total score $\geq 13, N=57$ ) with those of non-distressed parents (HADS total score $\leq 12, N=56$ ). One month after diagnosis, clinically distressed parents had more cognitions of Helplessness $(M=13.05$ vs. $M=10.41, F=19.96, p<.001, d=0.78)$ and fewer cognitions of Acceptance $(M=15.23$ vs. $M=18.91, F=$ $28.04, p<.001, d=0.89)$ than non-distressed parents. No significant results were found for Perceived Benefits. 
Table 2 Principal component analysis with oblique rotation and three fixed factors in parents of a child recently diagnosed with cancer $(N=242)$ and means and standard deviations, eigenvalues, \% variance explained, and Cronbach's alpha

Item

$$
\text { Rotated factor loadings }
$$

Helplessness Acceptance Perceived

Benefits

15. My child's illness frequently makes me feel helpless.

12. My child's illness limits me in everything that is important to me.

5. My child's illness controls my life.

1. Because of my child's illness, I miss the things I like to do most.

9. My child's illness prevents me from doing what I would really like to do.

7. My child's illness makes me feel useless at times.

10. I have learned to accept the limitations imposed by my child's illness.

3. I have learned to live with my child's illness.

13. I can accept my child's illness well.

17. I can cope effectively with my child's illness.

2. I can handle the problems related to my child's illness.

14. I think I can handle the problems related to my child's illness, even if the illness gets worse.

4. Dealing with my child's illness has made me a stronger person.

6. I have learned a great deal from my child's illness.

18. My child's illness has taught me to enjoy the moment more.

8. My child's illness has made life more precious to me.

16. My child's illness has helped me realize what is important in life.

11. Looking back, I can see that my child's illness has also brought about some positive changes in my life.

$M(\mathrm{SD})$

Eigenvalues

$\%$ of variance

$\alpha$

\begin{tabular}{|c|c|c|}
\hline .37 & -.40 & .28 \\
\hline .80 & -.03 & -.08 \\
\hline .55 & -.28 & .07 \\
\hline .86 & .14 & -.11 \\
\hline .87 & .04 & -.08 \\
\hline .48 & -.05 & .18 \\
\hline-.07 & .65 & .24 \\
\hline .00 & .83 & -.02 \\
\hline-.02 & .78 & -.04 \\
\hline .05 & .85 & .10 \\
\hline-.06 & .76 & -.02 \\
\hline .03 & .81 & -.08 \\
\hline-.03 & .47 & .55 \\
\hline .14 & .16 & .65 \\
\hline-.03 & -.01 & .79 \\
\hline .06 & -.08 & .82 \\
\hline-.04 & -.19 & .84 \\
\hline-.18 & .16 & .63 \\
\hline $12.45(3.31)$ & $16.60(3.93)$ & $15.88(4.04)$ \\
\hline 1.77 & 5.65 & 3.22 \\
\hline 9.83 & 31.38 & 17.90 \\
\hline .80 & .88 & .83 \\
\hline
\end{tabular}

\section{Discussion}

The results of this study confirm that the ICQ-P, which was originally developed for adult patients with a chronic disease, is suitable for assessing the cognitions of the parents of a child recently diagnosed with cancer. The same three-factor (each with 6 items) structure of the original ICQ (Helplessness, Acceptance, Perceived Benefits) was also found in two samples of parents of children recently diagnosed with cancer. Factor loadings were adequate and exceeded previously determined factor loadings, showing that the adapted ICQ (ICQ-P) had an adequate factor structure. All subscales showed high reliability $(\alpha=.80-.88)$, comparable to that of the original questionnaire [5]. Correlation analysis of the subscales of the ICQ-P demonstrated no association between Helplessness and Perceived Benefits, a small association between Acceptance and Perceived Benefits, and a moderate association between Helplessness and Acceptance, indicating that these factors are distinct constructs of illness cognitions.

The scores of mothers and fathers were not significantly different, consistent with an earlier study using the ICQ [5], but not with an earlier study of the parents of a child with cancer in which the Control Strategy Scale (CSS) was used [22]. Grootenhuis et al. investigated differences in control strategies in dyadic couples, and this might explain the absence of an effect in the present study. We used data obtained from one parent per child and therefore compared the scores of mothers and fathers of different families. Parental ICQ scores were not significantly different by cancer diagnosis - the parents of children with hematological, solid, or neurooncological tumors had similar beliefs of Helplessness, Acceptance, and Perceived Benefits. This suggests that illness cognitions are not determined by diagnosis, which was also found in a previous study [12]. Treatment intensity or survival perspective might be of influence, as suggested by a previous research [36], but we were not able to investigate this in the present study.

We found that parental illness cognitions were significantly correlated with psychological distress, with parents with beliefs of helplessness and little acceptance having a worse psychological well-being, in terms of overall distress, depression, anxiety, tension, irritation, and fatigue, than parents without 
Table 3 Correlations between the Illness Cognition Questionnaireparent version scales and measures of psychological distress

\begin{tabular}{llll}
\hline & Helplessness & Acceptance & Perceived Benefits \\
& $r$ & $r$ & $r$ \\
\hline Parental psychological well-being $^{*}$ & & \\
HADS Total $^{\mathrm{a}}$ & $.46^{* *}$ & $-.53^{* *}$ & -.06 \\
HADS Depression $^{\mathrm{a}}$ & $.42^{* *}$ & $-.49^{* *}$ & -.10 \\
HADS Anxiety $^{\mathrm{a}}$ & $.45^{* *}$ & $-.52^{* *}$ & -.02 \\
POMS Tension $^{\mathrm{b}}$ & $.42^{* *}$ & $-.39^{* *}$ & .03 \\
POMS Depression $^{\mathrm{b}}$ & $.59^{* *}$ & $-.54^{* *}$ & .02 \\
POMS Irritation $^{\mathrm{b}}$ & $.54^{* *}$ & $-.33^{* *}$ & .09 \\
POMS Fatique $^{\mathrm{b}}$ & $.53^{* *}$ & $-.29^{*}$ & -.06 \\
POMS Vigor $^{\mathrm{b}}$ & $-.41^{* *}$ & $.40^{* *}$ & .12 \\
\hline
\end{tabular}

HADS Total $M=13.7, \mathrm{SD}=7.5$, range $=0-33$; HADS Depression $M=6.3$, $\mathrm{SD}=4.0$, range $=0-17$; HADS Anxiety $M=7.4, \mathrm{SD}=3.9$, range $=0-18$; POMS Tension $M=7.5, \mathrm{SD}=5.2$, range $=0-23$; POMS Depression $M=$ $8.9, \mathrm{SD}=6.3$, range $=0-26$; POMS Irritation $M=6.7, \mathrm{SD}=5.4$, range $=0$ 22; POMS Fatigue $M=8.8, \mathrm{SD}=5.7$, range $=0-24$; POMS Vigor $M=8.5$, $\mathrm{SD}=4.3$, range $=0-19$

HADS Hospital Anxiety and Depression Scale, POMS Profiles of Mood States

$* p<.01 ; * * p<.001$

${ }^{\mathrm{a}} N=113$

${ }^{\mathrm{b}} N=126$

these beliefs. In addition, clinically distressed parents had more helplessness cognitions and fewer acceptance cognitions than did their non-distressed counterparts. Although the data were not suitable for causal analyses, these results are in line with earlier reports on the effect of parental illness beliefs on adaptation [5, 10-12]. Perceived Benefits were not significantly associated with parental psychological distress. This lack of correlation has been reported in other studies, in which benefit was found to be associated with positive constructs, such as trait optimism, positive mood, higher quality of life, and positive reframing as coping mechanism [37,38], and not with distress. Another possible explanation for this lack of correlation is that we included only families in which the child had recently been diagnosed with cancer, so that survival would be of key importance, finding benefit might occur later [39]. Thus, while all subscales of the ICQ-P were reliable and valid in the acute phase after the diagnosis of childhood cancer, the subscale Perceived Benefits might be more relevant in a later stage of cancer treatment. Changes in illness cognitions during long-term adjustment to disease and their predictive contribution to adjustment processes might be a topic for future research in these patient and parent groups.

The study had a number of strong points. It had a large sample, a broad range of children ages, a heterogeneous group of childhood cancer diagnoses, and mothers and fathers as respondents. However, it also had a number of limitations. First, we included the parents of children with cancer from two different studies; however, both samples completed questionnaires in the period shortly after diagnosis. While different questionnaires were used in the two studies, which reduced the sample size for analyses, similar results were obtained for both samples, which strengthened our conclusions. Although we intended to recruit both mothers and fathers, mothers were overrepresented in both samples; however, there were sufficient fathers to compare the scores of mothers with fathers. Secondly, this questionnaire was designed for the parents of sick children, and this is the only study to validate it in this population. In adults, the ICQ has proven to be reliable and valid in diverse patient groups [5], and therefore this can be expected to be the case for pediatric populations as well. Illness cognitions are generic and unrelated to medical factors, and therefore the ICQ-P should be applicable for children with chronic or life-threatening illnesses [5]. Future research should confirm the utility of this questionnaire in other pediatric populations. Thirdly, patients with a neuro-oncological diagnosis were underrepresented and patients with hematological diseases, especially ALL, were overrepresented in our sample. This is because study 1 consisted solely of ALL patients and their parents. However, we found that cancer diagnosis did not affect parental ICQ-P scores. Our crosssectional design limits our ability to assess causal relations and the long-term effects of illness cognitions on family adjustment.

Overall, this study showed that this version of the ICQ adapted for the parents of children with a chronic illness, specifically cancer, is a reliable and valid tool to evaluate illness cognitions. Assessment of the illness cognitions of the parents of children diagnosed with cancer is clinically relevant, because it may be a predictor of psychological distress, as suggested by our findings. The availability of a short and valid parent illness cognition assessment tool makes it possible to target interventions for parents at risk of maladaptive cognitions regarding their child's illness. Such interventions have been found to improve parental outcomes and to diminish child adjustment problems [40, 41].

Acknowledgments This research was supported by the Dutch Childhood Oncology Group (SKION), The Hague, The Netherlands, and the Dutch Cancer Society (KWF), The Netherlands. The authors would like to thank these service providers along with the local physicians and study managers in all participating centers (Emma Children's Hospital Academic Medical Center Amsterdam, University Medical Center Groningen, Leiden University Medical Center, University Medical Center Utrecht, Radboud University Medical Center Nijmegen, and VU University Medical Center Amsterdam). Also, we wish to express our gratitude to all participating families for their kind cooperation.

Conflict of interest The authors declare that they have no competing interests. 


\section{Appendix}

Illness Cognition Questionnaire-Parent version

\section{Instructions}

On the next page is a list of statements by parents of a chronically ill child. Please indicate the extent to which you agree with them by circling one of the answers following the statement. Do not spend too much time considering your answer. Your first impression is usually the best.

\section{To what extent do you agree with the following statements?}

Because of my child's illness, I miss the things I like to do most.

2. I can handle the problems related to my child's illness.

3. I have learned to live with my child's illness.

4. Dealing with my child's illness has made me a stronger person.

5. My child's illness controls my life.

6. I have learned a great deal from my child's illness.

7. My child's illness makes me feel useless at times.

8. My child's illness has made life more precious to me.

9. My child's illness prevents me from doing what I would really like to do.

10. I have learned to accept the limitations imposed by my child's illness.

11. Looking back, I can see that my child's illness has also brought about some positive changes in my life.

Not at all important to me.

13. I can accept my child's illness well.

14. I think I can handle the problems related to my child's illness, even if the illness get worse.

15. My child's illness frequently makes me feel helpless.

16. My child's illness has helped me realize what is important in life.

17. I can cope effectively with my child's illness.

18. My child's illness has taught me to enjoy the moment more.
1

1

1

1

1

1

1

1

1

1

som Somewhat To a large
extent

Completely

extent

2

3

4

$$
4
$$

$\begin{array}{llll}2 & 3 & 4\end{array}$

(1)

2

3

4

$\begin{array}{lll}2 & 3 & 4\end{array}$

$\begin{array}{llll}1 & 2 & 3 & 4\end{array}$

$\begin{array}{llll}1 & 2 & 3 & 4\end{array}$

$\begin{array}{llll}2 & 3 & 4\end{array}$

1

2

3

4

4

4

4

$\begin{array}{lll}1 & 2 & 3\end{array}$

$\begin{array}{llll}1 & 2 & 3 & 4\end{array}$

$\begin{array}{llll}1 & 2 & 3 & 4\end{array}$

$\begin{array}{llll}1 & 2 & 3 & 4\end{array}$

1

2

3

4

1

$2 \quad 3 \quad 4$


Open Access This article is distributed under the terms of the Creative Commons Attribution-NonCommercial 4.0 International License (http:// creativecommons.org/licenses/by-nc/4.0/), which permits any noncommercial use, distribution, and reproduction in any medium, provided you give appropriate credit to the original author(s) and the source, provide a link to the Creative Commons license, and indicate if changes were made.

\section{References}

1. Leventhal H (1986) Symptom reporting: a focus on process. In: McHugh S, Vallis TM (eds) Illness behavior: a multidisciplinary model. Plenum Press, New York, pp 219-237

2. Scharloo M, Kaptein AA (1997) Measurement of illness perceptions in patients with chronic somatic illness: a review. In: Petrie KJ, Weinman J (eds) Perceptions in health and illness: current research and applications. Harwood Academic Publishers, London, pp 103135

3. Kaptein AA, Broadbent E (2007) Illness cognition assessment. In: Ayers S (ed) Cambridge handbook of psychology, health and medicine. Cambridge University, Press, Cambridge, pp 268-273

4. Lazarus RS, Folkman S (1984) Appraisal, coping, and adaptational outcomes. In: Lazarus RS, Folkman S (eds) Stress, appraisal, and coping. Springer Publishing Company Inc., New York, pp 205-220

5. Evers AWM, Kraaimaat FW, Van Lankveld W, Jongen PJH et al (2001) Beyond unfavorable thinking: the illness cognition questionnaire for chronic diseases. J Consult Clin Psychol 69:10261036. doi:10.1037//0022-006X.69.6.1026

6. Heijmans M, de Ridder D (1998) Assessing illness representations of chronic illness: explorations of their disease-specific nature. $\mathrm{J}$ Behav Med 21:485-503

7. Scharloo M, Kaptein AA, Weinman J, Hazes JM et al (1998) Illness perceptions, coping and functioning in patients with rheumatoid arthritis, chronic obstructive pulmonary disease and psoriasis. J Psychosom Res 44:573-85

8. Hagger MS, Orbell S (2003) A meta-analytic review of the common-sense model of illness representations. Psychol Health 18:141-184. doi:10.1080/088704403100081321

9. Hudson JL, Bundy C, Coventry PA, Dickens C (2014) Exploring the relationship between cognitive illness representations and poor emotional health and their combined association with diabetes selfcare. A systematic review with meta-analysis. J Psychosom Res 76: 265-274. doi:10.1016/j.jpsychores.2014.02.004

10. Sloper P (2000) Predictors of distress in parents of children with cancer: a prospective study. J Pediatr Psychol 25:79-91. doi:10. 1093/jpepsy/25.2. 79

11. Stuber ML, Kazak AE, Meeske K, Barakat L et al (1997) Predictors of posttraumatic stress symptoms in childhood cancer survivors. Pediatrics 100:958-964. doi:10.1542/peds.100.6.958

12. Kazak AE, McClure KS, Alderfer MA, Hwang W et al (2004) Cancer-related parental beliefs: the Family Illness Beliefs Inventory (FIBI). J Pediatr Psychol 29:531-542. doi:10.1093/ jpepsy/jsh055

13. Grootenhuis MA, Last BF (1997) Adjustment and coping by parents of children with cancer: a review of the literature. Support Care Cancer 5:466-484

14. Currier JM, Hermes S, Phipps S (2009) Brief report: children's response to serious illness: perceptions of benefit and burden in pediatric cancer population. J Pediatr Psychol 34:1129-1134. doi: 10.1093/jpepsy/ jsp021

15. Fotiadou M, Barlow JH, Powell LA, Langton H (2008) Optimism and psychological well-being among parents of children with cancer: an exploratory study. Psycho Oncol 17:401-409. doi:10. 1002/pon. 1257

16. McCubbin M, Balling K, Possin P, Frierdich S et al (2002) Family resiliency in childhood cancer. Fam Relat 51:103-111

17. Robinson KE, Gerhardt CA, Vannatta K, Noll RB (2007) Parent and family factors associated with child adjustment to pediatric cancer. J Pediatr Psychol 32:400-410. doi:10.1093/jpepsy/js1038

18. Barrera M, Atenafu E, Hons JP (2009) Behavioral, social, and educational outcomes after pediatric stem cell transplantation and related factors. Cancer 115:880-889. doi:10.1002/cncr.24109

19. Colletti CJM, Wolfe-Christensen C, Carpentier M, Page MC et al (2008) The relationship of parental overprotection, perceived vulnerability, and parenting stress to behavioral, emotional, and social adjustment in children with cancer. Pediatr Blood Cancer 51:269274. doi: $10.1002 / p b c .21577$

20. Steele RG, Dreyer ML, Phipps S (2004) Patterns of maternal distress among children with cancer and their association with child emotional and somatic distress. J Pediatr Psychol 29:507-517

21. Stoppelbein L, Greening L, Wells H (2013) Parental coping and posttraumatic stress symptoms among pediatric cancer populations: tests of competing models. Psycho Oncol 22:2815-2822. doi:10. 1002/pon. 3358

22. Grootenhuis MA, Last BF, De Graaf-Nijkerk JH, Van der Wel M (1996) Secondary control strategies used by parents of children with cancer. Psycho Oncol 5:91-102

23. Verhoof EJA, Maurice-Stam H, Heymans HSA, Evers AWM et al (2014) Psychosocial well-being in young adults with chronic illness since childhood: the role of illness cognitions. Child Adolesc Psychiatr Ment Health 8:12

24. Van Litsenburg RRL, Huisman J, Pieters R, Verhaak C et al (2014) Determinants of quality of life during induction therapy in pediatric acute lymphoblastic leukemia. Support Care Cancer 22:32353242. doi:10.1007/s00520-014-2349-2

25. Van Tilburg CM, Sanders EAM, Nibbelke EE, Pieters R et al (2011) Impact of reduced chemotherapy treatment for good risk childhood acute lymphoblastic leukaemia on infectious morbidity. Br J Haematol 152:433-440. doi:10.1111/j.1365-2141.2010.08463

26. Evers AWM, Kraaimaat FW, van Lankveld W, Bijlsma JWJ (1998) De Ziekte Cognitie Lijst (ZCL). Gedragstherapie 31: 205-220

27. McNair DM, Lorr M, Droppleman LF (1971) Edits manual for the profile of mood states. Educational and Industrial Testing Service, San Diego

28. Wald FDM, Mellenbergh GJ (1990) De verkorte versie van de Nederlandse vertaling van de Profile of Moods States (POMS). Ned tijdschrift Psychologie 45:86-90

29. Zigmond AS, Snaith RP (1983) The hospital anxiety and depression scale. Acta Psychiatr Scand 67:361-370

30. Spinhoven P, Ormel J, Sloekers PP, Kempen GI et al (1997) A validation study of the Hospital Anxiety and Depression Scale (HADS) in different groups of Dutch subjects. Psychol Med 27: 363-370

31. Singer S, Kuhnt S, Götze H, Hauss J et al (2009) Hospital anxiety and depression scale cutoff scores for cancer patients in acute care. Br J Cancer 100:908-912. doi:10.1038/sj.bjc.6604952

32. Kaiser HF (1974) An index of factorial simplicity. Psychometrika 39:31-36

33. Stevens JP (2002) Applied multivariate statistics for the social sciences. Erlbaum, Hillsdale

34. Field A (2009) Discovering statistics using SPSS. SAGE Publications Ltd, London

35. Cohen JA (1992) Power primer. Psychol Bull 112:155-159

36. Grootenhuis MA, Last BF (2001) Children with cancer with different survival perspectives: defensiveness, control strategies, and psychological adjustment. Psycho Oncol 10:305-314 
37. Peterson C (2000) The future of optimism. Am Psychol 55:44-55. doi:10.1037/0003-066X.55.1.44

38. Urcuyo KR, Boyers AE, Carver CS, Antoni MH (2005) Finding benefit in breast cancer. Relations with personality, coping, and concurrent well-being. Psychol Health 20:175-192. doi:10.1080/ 08870440512331317634

39. Pascoe L, Edvardsson D (2013) Benefit finding in cancer: a review of influencing factors and health outcomes. Eur J Oncol Nurs 17: 760-766. doi:10.1016/ j.ejon.2013.03.005
40. Peek G, Mazurek Melnyk B (2014) A coping intervention for mothers of children diagnosed with cancer: connecting theory and research. Appl Nurs Res 27:202-204. doi:10.1016/j.apnr.2014.05. 002

41. Mazurek Melnyk B, Alpert-Gilles L, Fischbeck Feinstein N, Crean HF et al (2004) Creating opportunities for parent empowerment: program effects on the mental health/coping outcomes of critically ill young children and their mothers. Pediatrics 113:597-607 